\title{
EFFICACY OF CERTAIN ACARICIDES AGAINST TEGOLOPHUS GUAVAE AND BREVIPALPUS PHOENICIS ON GUAVA TREES
}

\author{
EL-HALAWANY, A. S. and K. M. EL-SAYED \\ Fruit Acarology, Plant Protection Research Institute, ARC, Dokki, Giza, Egypt.
}

(Manuscript received 22 July 2013)

\begin{abstract}
In the recent years Guava trees were infested with the rust mite Tegolophus guavae (Boczek) and Tenuipalpid mite Brevipalpus phoenicis (Geijskes) El-Halawany and Abou-Setta (2014). Inspection of guava trees showed that the population abundance of phytophagous mite, T. guavae was high on leaves and $B$. phoenicis was high on leaves and fruits. The present investigations were carried out to evaluate eight acaricides, (Agromic, Deva, Berfect, Nest, Baroq, Koncor, Menova and Ortus) against $T$. guavae and B. phoenicis under field conditions. Results indicated that Ortus, Menova, Agromic and Baroq were the most effective compounds in reducing guava rust mite population, $T$. guavae followed by Deva, Berfect and Koncor which gave good reduction over $(86.0 \%)$, while Nest was the lowest $(72 \%$ mortality). The reduction percentage of $B$. phoenicis after three weeks of application was $84 \%$ mortality, the highest reduction was recorded for Ortus $5 \%$ (90\%) on leaves and fruits.
\end{abstract}

\section{INTRODUCTION}

The guava rust mite $T$. guavae was recorded for the first time in Egypt on Psidium guajava L. by El-Halawany (2012) at Qalubia Governorate. After that ecological studies were done studied by (El-Halawany \& Abou-Setta, 2014). on guava indicated that $T$. guavae inhabiting leaves in February then increased in the number and reach maximum number in June and October especially on young leaves. On the other hand, the Tenuipalpid mite $B$. phoenicis inhabiting leaves in march and infested fruit after fruit setting and increased in the number in July on leaves and June on fruit causes brown-reddish. In Florida, USA, the mite T. guavae and Brevipalpus spp., cause damage to fruits and tender leaves (Pena et al., 1999). Highest numbers of $T$. guavae are observed in early autumn, winter and spring months. The mites are most often observed on fruit, causing deformations. The tenuipalpid mite, Brevipalpus phoenicis (Geijskes) is an important pest of more than 50 genera of ornamental plants, it's evidently well distributed throughout the world, this mite transmit virus leprosies when population are high (Jeppson et al., 1975), also Zaher (1984) found $B$. phoenicis is a principal of citrus, other hosts include guava, quince, sweet potato and acalypha, prefere the lower surfaces of leaves around the midrib and main veins, they were found also in pits, pedical and floral apex of fruits. 
Many researchers studied the effect of some acaricides against phytophagous mites. Rezk (1998) evaluated the two acaricides, Ortus (fenpyroximate) and Neoron (bromopropylate), on Brevipalpus obovatus on citrus orchards in Egypt.

On fig trees, abamectin showed a promising control against Tetranychus urticae, Rhyncophytoptus ficifoliae and Aceria ficus it caused reduction of $85 \%, 75 \%$ and $68 \%$ in population, respectively (Abou-Awad et al., 2000).

(Abou-Awad et al., (2005) showed the effect of abamectin on eriophyid olive mites, the results indicate that abamectin gave reduction of $85.40 \%$ and $88.90 \%$ in the population of Aceria oleae and Tegolophus hassani on leaves, respectively, during the 35-day period following applications. Similar results of abamectin biocide against eriophyid mites were found on citrus in Florida (Childers, 1986). Tayyib et al. (2005) in Pakistan evaluated new insecticides for controlling T. urticae on cotton. The chlorfenapyr gave highest mortality (87.5\%), also fenpyroximate gave (63.75\%), while the dicofol and azocyclotin gave less than $50 \%$ mortality.

AL-Joboory and AL-Jorany (2011) found that the efficacy some Acaricides (Envidor 240 SC, Ortus 5\% SC, Bye Bye 20 EC, King bow 24 ES and Abamectin 1.8\% EC) were a high sufficiency in controlling the mites and gave longer protection duration for the plants.

Al-Azzazy et al. (2013) indicated that the Abamectin was superior in reducing Aceria tulipae (79\% reduction) after 28 days.

This study aims to evaluate of certain acaricides for controlling T. guavae and $B$. phoenicis on Guava trees in Qalubia governorate.

\section{MATERIALS AND METHODS}

Eight of acaricides were evaluated on 17 May 2013 namely (Agromic 1.8\% EC, Deva 1.8 EW, Berfect 12\% EW, Nest 20\% SC, Baroq 10\% SC, Koncor 24\% SC, Menova $10 \% \mathrm{EC}$ and Ortus $5 \% \mathrm{SC}$ ). Two groves of guava trees were chosen one at Tanan village Qalubia Governorate, four acre, three years old which highly infested with Tegolophus guavae while, the other grove at El-saad village Qalubia Governorate, two acre seven years old which highly infested with Brevipalpus phoenicis, during season 2013. The two experiments design were randomized in complete block, included 180 trees. The two groves were divided into nine treatments were used including control, Each treatment included four replicates, each replicate represented with five trees. Each sample included 20 leaves and 10 fruits per replicate totally 80 leaves and 40 fruits per treatment after 3, 7, 14 and 21 days of each treatment to determine the reduction percentages. The compounds were sprayed by 
using a highly volume motor sprayer of 600 liters capacity. Pre-count was detected before spraying to estimate the reduction percentage in population by using (Henderson \& Tilton equation ,1955).

\section{Chemical acaricides:}

Table 1. Treatments and their application rates.

\begin{tabular}{|c|c|c|c|c|}
\hline Trt. & Trade name & Common name & Chemical name & $\begin{array}{l}\text { applicati } \\
\text { on/ } 100 \\
\text { liter of } \\
\text { water }\end{array}$ \\
\hline 1 & $\begin{array}{l}\text { Agromic } \\
1.8 \% \text { EC }\end{array}$ & Abamactin & $\begin{array}{l}\text { 5-O-demethylavermectin } A_{1 a}(i) \text { mixture with } \\
\text { 5-O-demethyl-25-de(1-methylpropyl)-25- (1- } \\
\text { methylethyl) avermactin } A_{1 a}(\text { ii) }\end{array}$ & $50 \mathrm{cc}$ \\
\hline 2 & Deva $1.8 \mathrm{EW}$ & Abamactin & $\begin{array}{l}\text { 5-O-demethylavermectin } A_{1 a}(i) \text { mixture with } \\
5 \text {-O-demethyl-25-de(1-methylpropyl)-25- (1- } \\
\text { methylethyl) avermactin } A_{1 a}(\mathrm{ii})\end{array}$ & $40 c c$ \\
\hline 3 & $\begin{array}{l}\text { Berfect } 12 \% \\
\text { EW }\end{array}$ & $\begin{array}{l}\text { 2\%Abamactin }+ \\
10 \% \text { Chlorofenapyr }\end{array}$ & $\begin{array}{l}\text { 5-O-demethylavermectin } \mathrm{A}_{1 a}(\mathrm{i}) \text { mixture with } \\
\text { 5-O-demethyl-25-de(1-methylpropyl)-25- (1- } \\
\text { methylethyl) avermactin } \mathrm{A}_{1 \mathrm{a}}(\mathrm{ii})+4 \text {-bromo-2- } \\
\text { (4-chlorophenyl)-1-ethoxymethyl-5- } \\
\text { trifluoromethyl-1H-pyrrole-3-carbonitrile }\end{array}$ & $30 c c$ \\
\hline 4 & Nest $20 \%$ SC & $\begin{array}{l}\text { 2\%Abamactin }+ \\
10 \% \text { Chlorofenapyr }\end{array}$ & $\begin{array}{l}\text { 5-O-demethylavermectin } \mathrm{A}_{1 \mathrm{a}}(\mathrm{i}) \text { mixture with } \\
\text { 5-O-demethyl-25-de(1-methylpropyl)-25- (1- } \\
\text { methylethyl) avermactin } \mathrm{A}_{1 \mathrm{a}}(\mathrm{ii})+4 \text {-bromo-2- } \\
\text { (4-chlorophenyl)-1-ethoxymethyl-5- } \\
\text { trifluoromethyl-1H-pyrrole-3-carbonitrile }\end{array}$ & $30 \mathrm{cc}$ \\
\hline 5 & $\begin{array}{l}\text { Baroq } 10 \% \\
\text { SC }\end{array}$ & Ectxazole & $\begin{array}{l}\text { 2-(2,6-difluorophenyl)-4-[4-(1,1- } \\
\text { dimethylethyl)-2-ethoxyphenyl]-4,5- } \\
\text { dihydrooxazole }\end{array}$ & $25 c c$ \\
\hline 6 & $\begin{array}{l}\text { Koncor } \\
24 \% S C\end{array}$ & Spirodiclofen & $\begin{array}{l}\text { 3-(2,4-dichlorophenyl)-2-oxo-1- } \\
\text { oxaspiro[4.5]dec-3-en-4-yl 2,2- } \\
\text { dimethylbutanoate }\end{array}$ & $40 c c$ \\
\hline 7 & $\begin{array}{l}\text { Menova } \\
10 \% \text { EC }\end{array}$ & Hexythiazox & $\begin{array}{l}\text { (4RS,5RS)-5-(4-chlorophenyl)- } N \text {-cyclohexyl-4- } \\
\text { methyl-2-oxo-1,3-thiazolidine-3-carboxamide }\end{array}$ & $50 \mathrm{cc}$ \\
\hline 8 & Ortus 5\%SC & fenpyroximate & $\begin{array}{l}\text { 1,1-dimethylethyl (E)-4- (((1.3-dimethyl-5- } \\
\text { Phenoxy-1 H- pyrazol-4-yl) methylen) amino) } \\
\text { methyl) benzoate. }\end{array}$ & $50 \mathrm{cc}$ \\
\hline
\end{tabular}

\section{Statistical analysis:}

Percent reduction of phytophagous mites were analyzed by two-way ANOVA and means were compared by using Fisher's least significant difference. Significance level was $P<0.05$. Analysis were conducted using SAS statistical software (SAS Institute, 2010). 


\section{RESULTS AND DISCUSSION}

The present study revealed that all tested acaricides were sufficient to control the guava rust mite, Tegolophus guavae and tenuipalpid mite, Brevipalpus phoenicis. As shown in Table (2) and Fig.(1) reduction percentage of guava eriophyid mite, $T$. guavae populations as a result of biocides and acaricides treatments under field conditions after 21 days. There were significant differences between acaricides exhibited reduction percentages. Ortus 5\% SC, Menova 10\% EC, Agromic 1.8\% EC and Baroq $10 \%$ SC the highest reduction percentage over $90 \%$ while, Deva $1.8 \%$, Berfect $12 \% \mathrm{EW}$, and Koncor $24 \%$ SC gave reduction over $85 \%$, the lowest reduction of population occurred for Nest $20 \%$ SC (72.24\%) after 21 days. Thus, it could be concluded that the all acaricides were effective against $T$. guavae.

Data in Table (3) and Fig.(2) cleared the effect of tested acaricides on tenuipalpid mite, B. phoenicis population on leaves. Ortus $5 \% \mathrm{SC}$ exhibited the highest reduction percentage, $93.63 \%$ followed by Deva $1.8 \%$ EW and Nest $20 \%$ SC $90 \%$. The reduction percentage of population for other treatments, 85.0, 87.94, 89.26, 86.62 and $88.5 \%$ for Agromic, Berfect, Baroq, Koncor and Menova, respectively.

On fruit, the fenpyroximate (Ortus) gave the highest reduction percentage (91.98\%), while non-significant different between Agromic, Deva, Berfect, Nest, Baroq, and Koncor, whereas, it's reduction percentage were 87.95, 85.79, 89.31, $89.75,89.36$ and $87.38 \%$ on $B$. phoenicis population after 21 days, respectively. Table (4) and Fig. (3). Menova also gave good reduction $84.28 \%$.

Obtained results indicated that all acaricides showed excellent results against the two phytophagous mites $T$. guavae and B. phoenicis under field conditions. Similar results were obtained by (Childer, 1986, Rezk, 1999, Pena, et al.,1999, Abou-Awad, et al., 2000, 2005, Tayyib, et al.,2005, Al-Azzazy et al., 2013, AL-Joboory and ALJorany, 2011). 


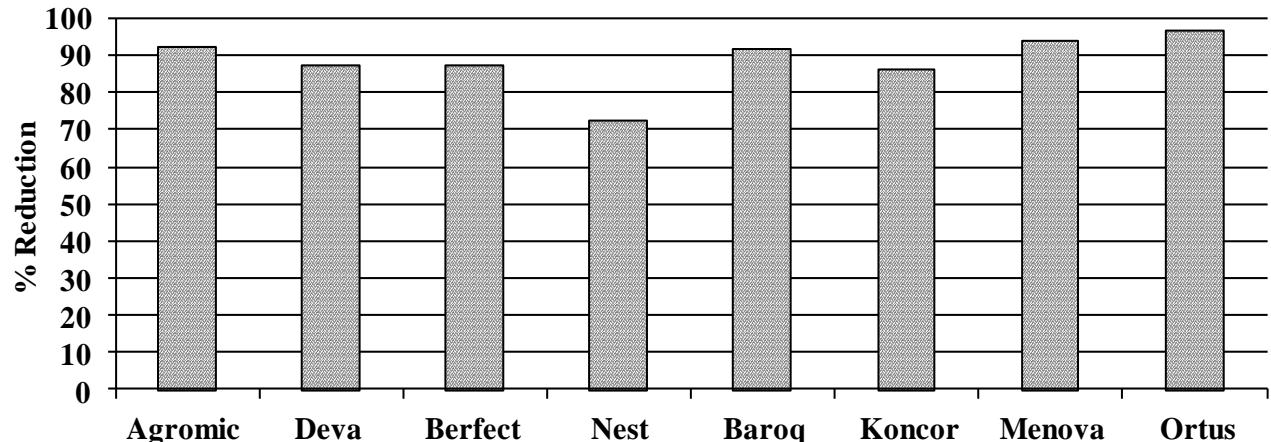

Acaricides

Fig. 1. Percentage reduction of guava rust mite $T$. guavae populations as a result of acaricides treatments under field conditions.

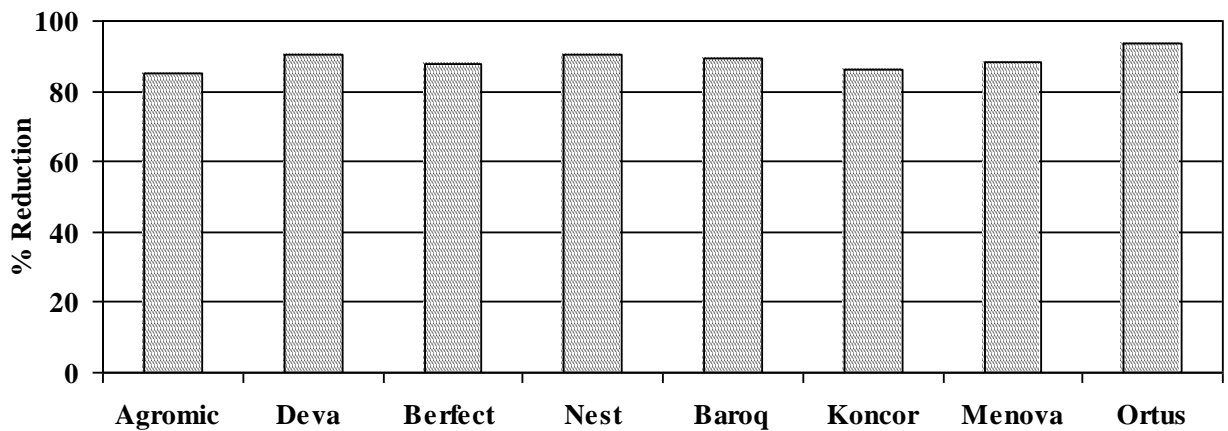

Acaricides

Fig. 2. Percentage reduction of tenuipalpid mite B. phoenicis populations on leaves as a result of acaricides tre atments under field conditions.

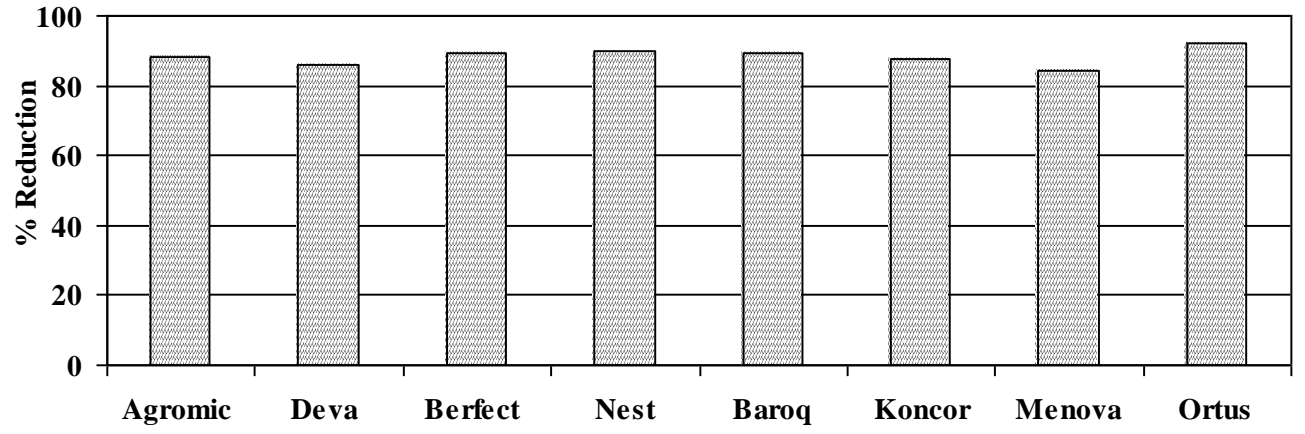

Acaricides

Fig. 3. Percentage reduction of tenuipalpid mite B. phoenicis populations on fruits as a result of acaricides treatments under field conditions. 
Table 2. Percentage reduction of guava rust mite $T$. guavae populations as a result of acaricides treatments under field conditions.

\begin{tabular}{|c|c|c|c|c|c|c|c|c|c|c|c|c|}
\hline \multirow{3}{*}{ Trade name } & \multirow{3}{*}{ Common name } & \multirow{3}{*}{$\begin{array}{c}\text { Rate of } \\
\text { application } \\
\text { / } 100 \text { Liter } \\
\text { of water }\end{array}$} & \multirow{3}{*}{$\begin{array}{l}\text { No. of } \\
\text { individuals } \\
\text { before } \\
\text { treatment }\end{array}$} & \multicolumn{8}{|c|}{ Number and \% reduction of individuals/ 80 leaves after treatment } & \multirow{3}{*}{$\begin{array}{c}\text { Average } \% \\
\text { reduction }\end{array}$} \\
\hline & & & & \multicolumn{2}{|c|}{3 days } & \multicolumn{2}{|c|}{ One week } & \multicolumn{2}{|c|}{ Two weeks } & \multicolumn{2}{|c|}{ Three weeks } & \\
\hline & & & & No. & $\%$ R. & No. & $\%$ R. & No. & $\%$ R. & No. & $\%$ R. & \\
\hline Ortus $5 \% \mathrm{SC}$ & fenpyroximate & $50 \mathrm{cc}$ & 27552 & 833 & 97.33 & 1112 & 96.60 & 1272 & 96.13 & 1620 & 95.81 & $96.47^{\mathrm{a}}$ \\
\hline Menova $10 \% \mathrm{EC}$ & Hexythiazox & $50 \mathrm{cc}$ & 27803 & 1594 & 94.94 & 1972 & 94.03 & 2164 & 93.48 & 2605 & 93.32 & 93.94 ab \\
\hline Agromic $1.8 \%$ EC & Abamactin & $50 \mathrm{cc}$ & 14792 & 958 & 94.28 & 1480 & 91.58 & 1514 & 91.42 & 1746 & 91.59 & $92.22^{b}$ \\
\hline Baroq $10 \%$ SC & Ectxazole & $25 c c$ & 11292 & 804 & 93.71 & 1156 & 91.38 & 1320 & 90.20 & 1494 & 90.58 & $91.47^{\mathrm{b}}$ \\
\hline Berfect $12 \%$ EW & $\begin{array}{l}\text { 2\%Abamactin }+ \\
10 \% \text { Chlorofenapyr }\end{array}$ & $30 \mathrm{cc}$ & 5916 & 578 & 91.37 & 1000 & 85.77 & 1133 & 83.94 & 1226 & 85.24 & $86.58^{c}$ \\
\hline Deva $1.8 \mathrm{EW}$ & Abamactin & $40 \mathrm{cc}$ & 5874 & 580 & 91.28 & 1000 & 85.67 & 1086 & 84.51 & 1140 & 86.18 & $86.91^{c}$ \\
\hline Koncor $24 \%$ SC & Spirodiclofen & $40 \mathrm{cc}$ & 7168 & 807 & 90.05 & 1123 & 86.80 & 1411 & 83.49 & 1637 & 83.72 & $86.02^{c}$ \\
\hline Nest $20 \%$ SC & $\begin{array}{l}2 \% \text { Abamactin }+ \\
10 \% \text { Chlorofenapyr }\end{array}$ & $30 \mathrm{cc}$ & 3934 & 652 & 85.36 & 1295 & 72.27 & 1642 & 65.02 & 1862 & 66.29 & $72.24^{d}$ \\
\hline Control & - & - & 24432 & 27666 & - & 29026 & - & 29156 & - & 34300 & - & - \\
\hline
\end{tabular}

No. =number $\quad$ R. =reduction $\quad L S D=4.19$ at level $5 \%$

Different letters in vertical column denote significant difference $(F$-test, $P<0.05, P<0.01)$. 
Table 3. Percentage reduction of tenuipalpid mite B. phoenicis populations on leaves as a result of acaricides treatments under field conditions.

\begin{tabular}{|c|c|c|c|c|c|c|c|c|c|c|c|c|}
\hline \multirow{3}{*}{ Trade name } & \multirow{3}{*}{ Common name } & \multirow{3}{*}{$\begin{array}{c}\text { Rate of } \\
\text { application } \\
\text { / } 100 \text { Liter } \\
\text { of water }\end{array}$} & \multirow{3}{*}{$\begin{array}{l}\text { No. of } \\
\text { individuals } \\
\text { before } \\
\text { treatment }\end{array}$} & \multicolumn{8}{|c|}{ Number and $\%$ reduction of individuals/ 80 leaves after treatment } & \multirow{3}{*}{$\begin{array}{l}\text { Average } \% \\
\text { reduction }\end{array}$} \\
\hline & & & & \multicolumn{2}{|c|}{3 days } & \multicolumn{2}{|c|}{ One week } & \multicolumn{2}{|c|}{ Two weeks } & \multicolumn{2}{|c|}{ Three weeks } & \\
\hline & & & & No. & $\%$ R. & No. & $\%$ R. & No. & $\%$ R. & No. & $\%$ R. & \\
\hline Ortus $5 \% \mathrm{SC}$ & fenpyroximate & $50 \mathrm{cc}$ & 691 & 24 & 96.65 & 38 & 94.99 & 47 & 87.59 & 105 & 87.59 & $93.36^{\mathrm{a}}$ \\
\hline Deva $1.8 \mathrm{EW}$ & Abamactin & $40 \mathrm{cc}$ & 784 & 61 & 92.49 & 74 & 91.41 & 78 & 87.18 & 123 & 87.18 & $90.66^{\mathrm{ab}}$ \\
\hline Nest $20 \%$ SC & $\begin{array}{l}2 \% \text { Abamactin }+ \\
10 \% \text { Chlorofenapyr }\end{array}$ & $30 \mathrm{cc}$ & 760 & 54 & 93.14 & 54 & 92.09 & 66 & 84.31 & 146 & 84.31 & $90.54^{\mathrm{ab}}$ \\
\hline Baroq $10 \%$ SC & Ectxazole & $25 c c$ & 802 & 52 & 93.74 & 66 & 92.51 & 117 & 83.19 & 165 & 83.19 & $89.26^{b c}$ \\
\hline Menova $10 \% \mathrm{EC}$ & Hexythiazox & $50 \mathrm{cc}$ & 525 & 31 & 94.30 & 40 & 93.06 & 91 & 81.33 & 120 & 81.33 & $88.50^{\mathrm{bcd}}$ \\
\hline Berfect $12 \%$ EW & $\begin{array}{l}2 \% \text { Abamactin }+ \\
10 \% \text { Chlorofenapyr }\end{array}$ & $30 \mathrm{cc}$ & 622 & 40 & 93.79 & 53 & 92.24 & 128 & 83.19 & 128 & 83.19 & $87.94^{\mathrm{bcd}}$ \\
\hline Koncor $24 \%$ SC & Spirodiclofen & $40 c c$ & 561 & 37 & 93.63 & 48 & 92.21 & 137 & 81.36 & 128 & 81.36 & $86.62^{\mathrm{cd}}$ \\
\hline Agromic $1.8 \%$ EC & Abamactin & $50 \mathrm{cc}$ & 509 & 49 & 90.70 & 62 & 88.91 & 95 & 76.25 & 148 & 76.25 & $85.00^{d}$ \\
\hline Control & - & - & 763 & 790 & - & 838 & - & 899 & - & 934 & - & - \\
\hline
\end{tabular}

No. =number $\quad$ R. $=$ reduction $\quad L S D=3.76$ at level $5 \%$

Different letters in vertical column denote significant difference (F-test, $\mathrm{P}<0.05, \mathrm{P}<0.01)$. 
Table 4. Percentage reduction of tenuipalpid mite B. phoenicis populations on fruits as a result of acaricides treatments under field conditions.

\begin{tabular}{|c|c|c|c|c|c|c|c|c|c|c|c|c|}
\hline \multirow{3}{*}{ Trade name } & \multirow{3}{*}{ Common name } & \multirow{3}{*}{$\begin{array}{c}\text { Rate of } \\
\text { application } \\
\text { / } 100 \text { Liter } \\
\text { of water }\end{array}$} & \multirow{3}{*}{$\begin{array}{c}\text { No. of } \\
\text { individuals } \\
\text { before } \\
\text { treatment }\end{array}$} & \multicolumn{8}{|c|}{ Number and $\%$ reduction of individuals/ 40 fruits after treatment } & \multirow{3}{*}{$\begin{array}{l}\text { Average } \% \\
\text { reduction }\end{array}$} \\
\hline & & & & \multicolumn{2}{|c|}{3 days } & \multicolumn{2}{|c|}{ One week } & \multicolumn{2}{|c|}{ Two weeks } & \multicolumn{2}{|c|}{ Three weeks } & \\
\hline & & & & No. & $\%$ R. & No. & $\% \mathrm{R}$. & No. & $\%$ R. & No. & $\%$ R. & \\
\hline Ortus $5 \% \mathrm{SC}$ & fenpyroximate & $50 \mathrm{cc}$ & 404 & 12 & 97.15 & 32 & 92.80 & 43 & 90.88 & 64 & 87.09 & $91.98^{\mathrm{a}}$ \\
\hline Nest $20 \%$ SC & $\begin{array}{l}\text { 2\%Abamactin }+ \\
10 \% \text { Chlorofenapyr }\end{array}$ & $30 \mathrm{cc}$ & 384 & 25 & 93.74 & 35 & 91.71 & 54 & 87.96 & 68 & 85.57 & $89.75^{\mathrm{ab}}$ \\
\hline Baroq $10 \%$ SC & Ectxazole & $25 c c$ & 442 & 29 & 93.69 & 40 & 91.77 & 61 & 88.18 & 88 & 83.77 & $89.36^{\mathrm{ab}}$ \\
\hline Berfect $12 \%$ EW & $\begin{array}{l}2 \% \text { Abamactin }+ \\
10 \% \text { Chlorofenapyr }\end{array}$ & $30 \mathrm{cc}$ & 336 & 24 & 93.14 & 31 & 91.61 & 46 & 88.27 & 65 & 84.23 & $89.31^{\mathrm{ab}}$ \\
\hline Agromic $1.8 \%$ EC & Abamactin & $50 \mathrm{cc}$ & 226 & 13 & 94.47 & 21 & 91.55 & 37 & 85.98 & 56 & 79.81 & $87.95^{\mathrm{bc}}$ \\
\hline Koncor $24 \%$ SC & Spirodiclofen & $40 \mathrm{cc}$ & 265 & 14 & 94.92 & 27 & 90.74 & 49 & 84.16 & 66 & 79.70 & $87.38^{\mathrm{bc}}$ \\
\hline Deva $1.8 \mathrm{EW}$ & Abamactin & $40 \mathrm{cc}$ & 196 & 14 & 93.14 & 20 & 90.72 & 36 & 84.27 & 60 & 75.05 & $85.79^{\mathrm{bc}}$ \\
\hline Menova $10 \%$ EC & Hexythiazox & $50 \mathrm{cc}$ & 245 & 15 & 94.12 & 20 & 92.58 & 59 & 79.37 & 87 & 71.06 & $84.28^{\mathrm{c}}$ \\
\hline Control & - & - & 370 & 385 & - & 407 & - & 432 & - & 454 & - & - \\
\hline
\end{tabular}

No. =number $\quad$ R. $=$ reduction $\quad L S D=3.95$ at level $5 \%$

Different letters in vertical column denote significant difference ( $F$-test, $\mathrm{P}<0.05, \mathrm{P}<0.01$ ). 


\section{REFERENCES}

1. Abou-Awad, B. A. El-Sawaf, B. M. Reda, A. S. and A. A. Abdel-Khalek. 2000. Environmental management and biological aspects of two eriophyoid fig mites in Egypt: Aceria ficus and Rhyncophytoptus ficifoliae. Acarologia, 40: 419-429.

2. Abou-Awad, B. A., A. M. Metwally and M. M. Al-Azzazy. 2005. Environmental management and biological aspects of two Eriophyid olive mites in Egypt: Aceria oleae (Nalepa) and Tegolophus hassani (Keifer). Z. Pflanzenkrankh. Pflanzensch., 112 (3): 287-303.

3. Al-Azzazy, M.M., A.A. Abdallaha and H.M. El-Kawas. 2013. Studies on the wheat curl mite, Aceria tulipae Keifer (Eriophyidae), in Egypt. Arch. of Phytopathol. Plant Protection, 46 (10): 1150-1158.

4. AL-Joboory, H. K. and R. S. AL-Jorany. 2011. Effect of infection by the olive bud mite Aceria oleae (Nalepa) and the olive rust mite Tegolophus hassani (Keifer) on some characters growth vegetation of olive nurseries and the efficacy control of some acaricides on their. Diyala J. of Agric. Sci., 3 (2): 364-372.

5. Childers C.C. 1986. Methods for the routine screening of acaricides against the citrus rust mite Phyllocoptruta oleivora (Ashmead) ( Acari: Eriophyidae). British Crop Protection conf., Pests and Diseases 3, C-17.

6. El-Halawany, A.S.H. 2012. Survey of eriophyid mites on some fruit trees, with redescriptions of two newly recorded species and a checklist of eriophyid mites in Egypt (Acari: Eriophyoidea). Egypt. Acad. J. biolog. Sci., 5 (2):205 -216.

7. El-Halawany, A.S. and M. M. Abou-Setta. 2014. Mites inhibiting guava trees and their dynamics in relation to weather factors and plant phenology. Acarines (In press).

8. Henderson, C. and E. Tilton. 1955. Test with acaricides against the brown wheat mite. J. Econ. Entomol., 84:157-161.

9. Jeppson, L. R., H. H. Keifer and E. W. Baker. 1975. Mites injurious to economic plants. University of California Press, California, $614 \mathrm{pp}$.

10. Pena, J. E., R. Duncan, and T.Vasquez. 1999. Guava arthropod seasonality and control of fruit files in South Florida. Proc. Fla. State Hort. Soc., 112: 206-209.

11. Rezk, H. A. 1998. The false spider mite, Brevipalpus obovatus Donnadieu (Acari: Tenuipalpidae): host-related biology, seasonal abundance, and control. Acarology: Proceedings of the 10th International Congress, Canbrra, Australia. 291-294.

12. SAS Institute. 2010. SAS Statistics and Graphics Guide, Release 9.1. SAS Institute, Cary, North Carolina 27513, USA.

13. Tayyib, M., A.S. Sohail, A. Murtaza and F.F. Jamil. 2005. Efficacy of some newchemistry insecticides for controlling the sucking insect pests and mites on cotton. Pakistan Entomol. Vol. 27 (1): 63-66.

14. Zaher, M. A. 1984. Survey and ecological studies on phytophagous, predaceous and soil mites in Egypt. I: Phytophagous mites in Egypt (Nile vally and Delta). PL.480 Programme. USA Project No. EG. ARS, 30. Grant. No, FG, Eg., 1-181pp. 
كفاءة بعض المبيدات الأكاروسية لمكافحه الحلم الدودي Tegolophus guavae والأكاروس المبطb Brevipalpus phoenicis على أثجار الجوافة

$$
\begin{aligned}
& \text { أثرف سعيد الحلوانى، كرم السيد محمد } \\
& \text { أكاروس الفاكهة - معهُ بحوث وقاية النباتات - مركز البحوث الزراعية- الدقي- جيزة }
\end{aligned}
$$

في السنوات الأخيرة ظهرت على أثجار الجو افة أعر اض صدأ على الأوراق نتيجــة إصــابتها Cالحلم الدوى Tegolophus guavae (Boczek و الحلم المبطط

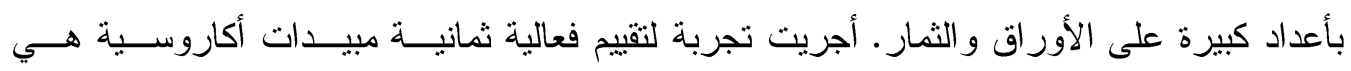

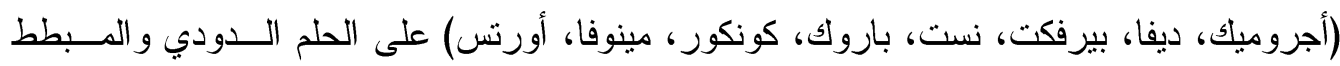

$$
\text { تحت الظروف الحقلية. }
$$

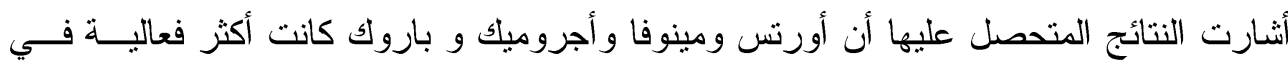

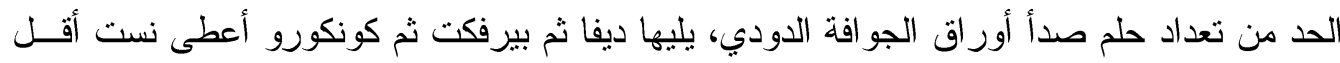

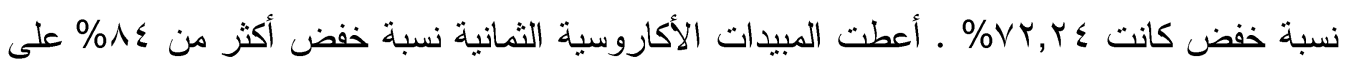

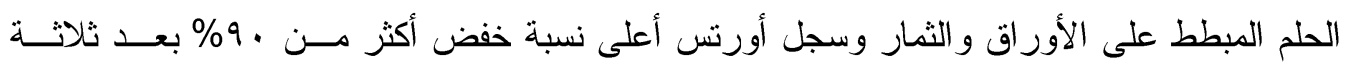
أسابيع من الرش.

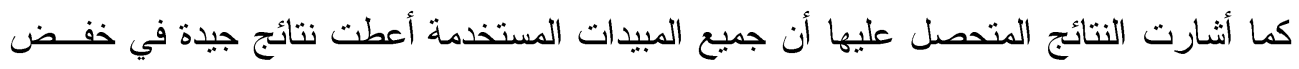
تعداد كل من الحلم الدودي والأكاروس المبطط على أشجار الجو افة تحت الظروف الحقلية. 